\title{
An efficient algorithm to compute transient pressure responses of slanted wells with arbitrary inclination in reservoirs
}

\author{
Wang Haitao $^{1 *}$, Zhang Liehui ${ }^{1}$, Guo Jingjing ${ }^{1}$, Liu Qiguo ${ }^{1}$ and He Xinming ${ }^{2}$ \\ ${ }^{1}$ State Key Laboratory of Oil and Gas Reservoir Geology and Exploitation, Southwest Petroleum University, Chengdu, \\ Sichuan 610500, China \\ ${ }^{2}$ Research Institute of Petroleum Exploration \& Production, Sinopec Northwest Company, Urumqi, Xinjiang 830011, China
}

(C) China University of Petroleum (Beijing) and Springer-Verlag Berlin Heidelberg 2012

\begin{abstract}
Compared with vertical and horizontal wells, the solution and computation of transient pressure responses of slanted wells are more complex. Vertical and horizontal wells are both simplified cases of slanted wells at particular inclination, so the model for slanted wells is more general and more complex than other models for vertical and horizontal wells. Many authors have studied unsteady-state flow of fluids in slanted wells and various solutions have been proposed. However, until now, few of the published results pertain to the computational efficiency. Whether in the time domain or in the Laplace domain, the computation of integration of complex functions is necessary in obtaining pressure responses of slanted wells, while the computation of the integration is complex and time-consuming. To obtain a perfect type curve the computation time is unacceptable even with an aid of high-speed computers. The purpose of this paper is to present an efficient algorithm to compute transient pressure distributions caused by slanted wells in reservoirs. Based on rigorous derivation, the transient pressure solution for slanted wells of any inclination angle is presented. Assuming an infinite-conductivity wellbore, the location of the equivalent-pressure point is determined. More importantly, according to the characteristics of the integrand in a transient pressure solution for slanted wells, the whole integral interval is partitioned into several small integral intervals, and then the method of variable substitution and the variable step-size piecewise numerical integration are employed. The amount of computation is significantly reduced and the computational efficiency is greatly improved. The algorithm proposed in this paper thoroughly solved the difficulty in the efficient and high-speed computation of transient pressure distribution of slanted wells with any inclination angle.
\end{abstract}

Key words: Arbitrary inclination, slanted well, transient pressure behavior, efficient algorithm, variable step-size, piecewise integration

\section{Introduction}

Many authors have studied unsteady-state flow of fluids in slanted wells and various solutions have been proposed. Early in the 1970s, Cinco et al (Cinco, 1974; Cinco et al, $1975 a ; 1975 b)$ investigated the unsteady-state pressure distribution created by a slanted well and presented a solution in real space. However, all their derivations are done in real space and the computation of transient pressure distribution according to the final solution is time-consuming. Moreover, the effects of wellbore storage and skin factor could not be easily incorporated in the solution. Besson (1990) studied the performance of horizontal and slanted wells in anisotropic reservoirs. Khattab et al (1991) presented pressure transient

*Corresponding author. email: wanghaitao1999_@163.com

Received June 21, 2011 responses for single and multiple-layered reservoirs. Chen et al (1995) investigated the pressure distribution created by a slanted well with elliptical inner boundary conditions. $\mathrm{Lu}$ (1997) studied the transient pressure behavior of slanted and horizontal wells in layered reservoirs with crossflow. Various solutions for pressure responses of slanted wells were proposed in the published literature. However, as pointed out by Ozkan and Raghavan (2000), all the solutions are not particularly useful because of numerical difficulties. They presented a new computationally efficient solution to compute pressure distributions caused by inclined or slanted wells, but they only presented the final forms of the expression and did not provide the derivation process. What is more, the computational efficiency of their new solution sometimes is not so satisfactory, and even big errors might occur when it is applied to calculating limiting cases. Harmohan et al (2007) designed numerical simulation studies to investigate 
the pressure transient behavior of horizontal and slant wells that intersect high permeability layers sandwiched between two lower permeability layers. Fair (2008) studied pressure transient behavior of horizontal wells by adapting the slant well solution to layered media. However, the numerical difficulties still had not been solved successfully.

In addition to the above, some Chinese authors also have studied the pressure transient behavior of slanted wells. Liao (1998) proposed a mathematical model to describe the pseudo steady state flow toward a slanted well in dual porosity reservoirs. Wang et al (2005) pointed out that the pressure response of an inclined multiwell system can be obtained by superposing the pressure response of each inclined well in different coordinate systems, and they investigated the pressure distribution of an inclined multiwell system based on this method. In 2006, Wang et al (2006a; 2006b) discussed the computational efficiency and the multi-solution feature of the slanted well test model. Li et al (2009) studied the pressure transient behavior of inclined wells with complex boundaries based on the source function and the Newman product method.

It can be recognized that most authors mainly concentrate on the construction of various well test models and neglect the practical problem when applying theoretical models to field production, i.e. the computational efficiency of the traditional algorithm is low and the accuracy is poor.

The focus of this work is on computational issues. Based on rigorous derivation, a transient pressure solution for slanted wells is presented. Assuming an infinite-conductivity wellbore, the location of the equivalent-pressure point is determined. More importantly, according to the characteristics of the integrand in a transient pressure solution for slanted wells, the whole integral interval is partitioned into several small integral intervals, and then the methods of variable substitution and variable step-size piecewise numerical integration are employed. The amount of computation is greatly reduced and the computational efficiency is significantly improved. The algorithm proposed in this paper thoroughly solves the difficulty in the efficient and high-speed computation of transient pressure distribution of slanted wells.

\section{Basic assumptions and mathematical model for slanted wells}

Fig. 1 shows a schematic for a slanted well in a laterally infinite reservoir. The top and bottom boundaries are impermeable. The formation thickness is $h . k_{\mathrm{h}}$ and $k_{\mathrm{v}}$ represent the permeability in the horizontal and vertical directions, respectively. The length of the slanted well is $L_{\mathrm{w}}$. The definition of the coordinate system is shown in Fig. 1. The axis of the slanted well is in the $X-Z$ plane. The midpoint of the slanted well is on the $Z$ axis. The inclination angle of the well, $\theta$, is measured from the positive $Z$-axis in the anticlockwise direction. The midpoint of the slanted well is located at an elevation $z_{\mathrm{wm}}$.

The basic assumptions are as follows:

1) The slanted well produces at a constant rate $q$;

2) Single phase flow of slightly compressible fluid, with a



Fig. 1 Schematic of the slanted well model with impermeable top and bottom boundaries

constant compressibility;

3) Darcy flow in the reservoir;

4) The pressure is uniform initially throughout the reservoir and is equal to $p_{\mathrm{i}}$;

5) Gravitational and capillary effects are negligible.

\section{Transient pressure responses of slanted wells in anisotropic reservoirs}

\subsection{Continuous point-source solution in anisotropic reservoirs}

Ozkan and Raghavan (1991a; 1991b) derived the instantaneous point-source solution for infinite porous media in the Laplace domain with the generalized Dirac delta function. Based on the result, the continuous point-source solutions for infinite porous media in the Laplace domain could be obtained by applying the principle of superposition. Then the method of images and Poisson's summation formula were applied to getting the continuous point-source solutions for laterally infinite reservoirs with impermeable top and bottom boundaries. The continuous point-source solution is given as follows:

$$
\Delta \bar{p}=\frac{\overline{\hat{q}} B \mu}{2 \pi k_{\mathrm{h}} h}\left[K_{0}\left(r_{\mathrm{D}} \sqrt{s}\right)+2 \sum_{n=1}^{\infty} K_{0}\left(r_{\mathrm{D}} \alpha_{n}\right) \cos \beta_{n} \cos \varepsilon_{n}\right]
$$

The terms in Eq. (1) are defined as follows:

$$
\begin{aligned}
& r_{\mathrm{D}}=\sqrt{\left(x_{\mathrm{D}}-x_{\mathrm{wD}}\right)^{2}+\left(y_{\mathrm{D}}-y_{\mathrm{wD}}\right)^{2}}, \quad h_{\mathrm{D}}=\frac{h}{L} \sqrt{\frac{k_{\mathrm{h}}}{k_{\mathrm{v}}}}, \\
& \alpha_{n}=\sqrt{s+\frac{n^{2} \pi^{2}}{h_{\mathrm{D}}^{2}}}, \quad \beta_{n}=n \pi \frac{z_{\mathrm{D}}}{h_{\mathrm{D}}}, \quad \varepsilon_{n}=n \pi \frac{z_{\mathrm{wD}}}{h_{\mathrm{D}}}, \\
& x_{\mathrm{D}}=\frac{x}{L}, \quad x_{\mathrm{wD}}=\frac{x_{\mathrm{w}}}{L}, \quad y_{\mathrm{D}}=\frac{y}{L}, \quad y_{\mathrm{wD}}=\frac{y_{\mathrm{w}}}{L}, \\
& z_{\mathrm{D}}=\frac{z}{L} \sqrt{\frac{k_{\mathrm{h}}}{k_{\mathrm{v}}}}, \quad z_{\mathrm{wD}}=\frac{z_{\mathrm{w}}}{L} \sqrt{\frac{k_{\mathrm{h}}}{k_{\mathrm{v}}}}
\end{aligned}
$$

where $k_{\mathrm{h}}$ is the permeability in the horizontal direction, $\mathrm{m}^{2}$; $k_{\mathrm{v}}$ is the permeability in the vertical direction, $\mathrm{m}^{2} ; L$ is the 
reference length of the system, and here $L=0.5 L_{\mathrm{w}}, \mathrm{m} ; \overline{\hat{q}}$ is the Laplace transform of $\hat{q}$; $\hat{q}$ is the production rate from the continuous point source, $\mathrm{m}^{3} / \mathrm{s} ; \Delta \bar{p}$ is the Laplace transform of $\Delta p$ with respect to the dimensionless time $t_{\mathrm{DL}}$, and its definition is given in Eq. (2), $\mathrm{Pa}$; $t_{\mathrm{DL}}$ is the dimensionless time and its definition is given in Eq. (3), dimensionless; $\Delta p$ is the pressure difference in the reservoir, i.e., $\Delta p=p_{\mathrm{i}}-p, \mathrm{~Pa} ; p_{\mathrm{i}}$ is the initial reservoir pressure, $\mathrm{Pa} ; B$ is the formation volume factor, dimensionless; $\mu$ is the oil viscosity, $\mathrm{Pa} \cdot \mathrm{s} ; h$ is the formation thickness, $\mathrm{m}$; $s$ is the Laplace transform variable with respect to $t_{\mathrm{DL}}$, dimensionless; $x, y$ and $\mathrm{z}$ are distances in the $x, y$ and $z$ directions, respectively, $\mathrm{m} ; x_{\mathrm{w}}, y_{\mathrm{w}}$ and $z_{\mathrm{w}}$ are the locations of the point source in the $x, y$ and $z$ directions, respectively, $\mathrm{m}$; $z_{\mathrm{wm}}$ is the location of the well center (midpoint) in the vertical direction, $\mathrm{m}$.

The Laplace transform of $\Delta p$ is given as follows:

$$
\Delta \bar{p}=\int_{0}^{+\infty} \Delta p \exp \left(-s t_{\mathrm{DL}}\right) \mathrm{d} t_{\mathrm{DL}}
$$

The dimensionless time $t_{\mathrm{DL}}$ is defined as follows:

$$
t_{\mathrm{DL}}=\frac{k_{\mathrm{h}} t}{\phi \mu C_{\mathrm{t}} L^{2}}
$$

where $t$ is the production time, $\mathrm{s} ; \phi$ is the porosity, fraction; $C_{\mathrm{t}}$ is the total compressibility, $\mathrm{Pa}^{-1}$.

\subsection{Slanted line-source solution in anisotropic reservoirs}

The slanted well in Fig. 1 can be assumed as a slanted line-source well. The transient pressure response corresponding to the fluid withdrawal from a line-source can be obtained by integrating Eq. (1) along the axis of the slanted well. Thus, we will have

$$
\Delta \bar{p}=\int_{l} \frac{\overline{\tilde{q}} B \mu}{2 \pi k_{\mathrm{h}} h}\left[K_{0}\left(r_{\mathrm{D}} \sqrt{s}\right)+2 \sum_{n=1}^{\infty} K_{0}\left(r_{\mathrm{D}} \alpha_{n}\right) \cos \beta_{n} \cos \varepsilon_{n}\right] \mathrm{d} l
$$

where $l$ is the integration path of line source; $\tilde{q}$ is the production rate per unit length, $\mathrm{m}^{3} /(\mathrm{s} \cdot \mathrm{m}) ; \overline{\tilde{q}}$ is the Laplace transform of $\tilde{q} ; \mathrm{d} l$ is the differential element of the line, $\mathrm{d} l=\sqrt{\left(\mathrm{d} x_{\mathrm{w}}\right)^{2}+\left(\mathrm{d} z_{\mathrm{w}}\right)^{2}}, \mathrm{~m}$.

The computation of the line integration in Eq. (4) is not convenient, and it can be reduced to the integration of $x_{\mathrm{w}}$ :

$$
\begin{aligned}
\Delta \bar{p} & =\int_{-\frac{L_{\mathrm{w}}}{2} \sin \theta}^{\frac{L_{\mathrm{w}}}{2} \sin \theta} \frac{\overline{\tilde{q}} B \mu}{2 \pi k_{\mathrm{h}} h} \sqrt{1+\left(\frac{\mathrm{d} z_{\mathrm{w}}}{\mathrm{d} x_{\mathrm{w}}}\right)^{2}} \\
& \times\left[K_{0}\left(\left(r_{\mathrm{D}} \sqrt{s}\right)+2 \sum_{n=1}^{\infty} K_{0}\left(r_{\mathrm{D}} \alpha_{n}\right) \cos \beta_{n} \cos \varepsilon_{n}\right] \mathrm{d} x_{\mathrm{w}}\right.
\end{aligned}
$$

$0<\theta<\pi / 2$ in the whole paper unless special statements are made.

Because $x_{\mathrm{wD}}=x_{\mathrm{w}} / L$, so Eq. (5) can be transformed into:

$$
\begin{aligned}
\Delta \bar{p} & =\int_{-\frac{L_{\mathrm{w}}}{2 L} \sin \theta}^{\frac{L_{\mathrm{w}}}{2 L} \sin \theta} \frac{\overline{\tilde{q}} B \mu L}{2 \pi k_{\mathrm{h}} h} \sqrt{1+\left(\frac{\mathrm{d} z_{\mathrm{w}}}{\mathrm{d} x_{\mathrm{w}}}\right)^{2}} \\
& \times\left[K_{0}\left(r_{\mathrm{D}} \sqrt{s}\right)+2 \sum_{n=1}^{\infty} K_{0}\left(r_{\mathrm{D}} \alpha_{n}\right) \cos \beta_{n} \cos \varepsilon_{n}\right] \mathrm{d} x_{\mathrm{wD}}
\end{aligned}
$$

It can be seen from Fig. 1 that on the inclined line source the following conditions must be satisfied:

$$
\begin{aligned}
& z_{\mathrm{w}}=z_{\mathrm{wm}}-x_{\mathrm{w}} \cot \theta \\
& y_{\mathrm{w}}=0
\end{aligned}
$$

where $z_{\mathrm{wm}}$ is the location of the well center in the vertical direction, $\mathrm{m}$.

In the distorted space, the dimensionless form of Eq. (7) is as follows:

$$
x_{\mathrm{wD}}=\tan \theta^{\prime}\left(z_{\mathrm{wD}}-z_{\mathrm{wmD}}\right)
$$

with

$$
\tan \theta^{\prime}=\sqrt{\frac{k_{\mathrm{v}}}{k_{\mathrm{h}}}} \tan \theta
$$

With Eq. (7), Eq. (6) can be changed into:

$$
\begin{aligned}
\Delta \bar{p} & =\int_{-\frac{L_{\mathrm{w}}}{2 L} \sin \theta}^{\frac{L_{\mathrm{w}}}{2 L} \sin \theta} \frac{\overline{\tilde{q}} B \mu L}{2 \pi k_{\mathrm{h}} h} \csc \theta \\
& \times\left[K_{0}\left(r_{\mathrm{D}} \sqrt{s}\right)+2 \sum_{n=1}^{\infty} K_{0}\left(r_{\mathrm{D}} \alpha_{n}\right) \cos \beta_{n} \cos \varepsilon_{n}\right] \mathrm{d} x_{\mathrm{wD}}
\end{aligned}
$$

Eq. (11) can be written in the following form:

$$
\Delta \bar{p}=\Delta \bar{p}_{1}+\Delta \bar{p}_{2}
$$

The expression of the first item $\Delta \bar{p}_{1}$ is:

$$
\Delta \bar{p}_{1}=\int_{-\frac{L_{\mathrm{w}}}{2 L} \sin \theta}^{\frac{L_{\mathrm{w}}}{2 L} \sin \theta} \frac{\overline{\tilde{q}} B \mu L}{2 \pi k_{\mathrm{h}} h} \csc \theta K_{0}\left(r_{\mathrm{D}} \sqrt{s}\right) \mathrm{d} x_{\mathrm{wD}}
$$

The expression of the second item $\Delta \bar{p}_{2}$ is:

$$
\Delta \bar{p}_{2}=\int_{-\frac{L_{\mathrm{w}}}{2 L} \sin \theta}^{\frac{L_{\mathrm{w}}}{2 L} \sin \theta} \frac{\overline{\tilde{q}} B \mu L}{\pi k_{\mathrm{h}} h} \csc \theta\left[\sum_{n=1}^{\infty} K_{0}\left(r_{\mathrm{D}} \alpha_{n}\right) \cos \beta_{n} \cos \varepsilon_{n}\right] \mathrm{d} x_{\mathrm{wD}}
$$

\subsection{Line source solution with uniform flux wellbore}

Theoretically speaking, it is more reasonable to treat the 
slanted wellbore as an infinite-conductivity line source than as a uniform flux line source. However, the wellbore pressure solution of an infinite-conductivity slanted line source cannot easily be obtained. Studies have shown that by choosing an appropriate point at which the wellbore pressure response is computed, the wellbore pressure solution of an infiniteconductivity line source can be approximated by the pressure solution of a uniform flux line source. So, we here first investigate the transient pressure solution of a uniform flux wellbore.

Assuming that the fluid withdrawal along the wellbore is uniformly distributed, we have

$$
\begin{aligned}
& \tilde{q}=\frac{q}{L_{\mathrm{w}}} \\
& \overline{\tilde{q}}=\frac{\tilde{q}}{s}=\frac{1}{s} \frac{q}{L_{\mathrm{w}}}
\end{aligned}
$$

Let $L=L_{\mathrm{w}} / 2$, and with Eqs. (8) and (16), Eq. (13) can be transformed into:

$$
\Delta \bar{p}_{1}=\frac{c_{1}}{2 s} \int_{-\sin \theta}^{\sin \theta} K_{0}\left(\sqrt{\left(x_{\mathrm{D}}-x_{\mathrm{wD}}\right)^{2}+y_{\mathrm{D}}^{2}} \sqrt{s}\right) \mathrm{d} x_{\mathrm{wD}}
$$

with

$$
c_{1}=\frac{q B \mu}{2 \pi k_{\mathrm{h}} h} \csc \theta
$$

Similarly, with Eqs. (8), (9) and (16), Eq. (14) is transformed into:

$$
\Delta \bar{p}_{2}=\frac{c_{1}}{s} \int_{-\sin \theta}^{\sin \theta}\left[\sum_{n=1}^{\infty} K_{0}\left(\xi\left(x_{\mathrm{wD}}, y_{\mathrm{D}}\right) \alpha_{n}\right) \cos \beta_{n} \cos \varepsilon_{n}\right] \mathrm{d} x_{\mathrm{wD}}
$$

with

$$
\begin{aligned}
& \xi\left(x_{\mathrm{wD}}, y_{\mathrm{D}}\right)=\sqrt{\left(x_{\mathrm{D}}-x_{\mathrm{wD}}\right)^{2}+y_{\mathrm{D}}^{2}} \\
& \varepsilon_{n}=n \pi \frac{\frac{x_{\mathrm{wD}}}{\tan \theta^{\prime}}+z_{\mathrm{wmD}}}{h_{\mathrm{D}}}
\end{aligned}
$$

Eqs. (12), (17) and (18) denote the pressure drawdown at any point in the reservoir created by a slanted well along which the fluid withdrawal is uniformly distributed. One can get the expression of transient pressure response of a slanted well in real space by the method of inversion. In real space the pressure dropdown is the function of space variables (i.e., $x_{\mathrm{D}}, y_{\mathrm{D}}$ and $z_{\mathrm{D}}$ ) and time $t_{\mathrm{D}}$.

\subsection{Bottom hole pressure of infinite-conductivity line source (inclined well)}

Theoretical analyses and practices have shown that the fluid flow in the slanted wellbore at the perforated interval is pipe flow, and the pressure drop in this process is negligible compared to that caused by fluid flow in the porous medium. That is the reason that we treat the slanted wellbore as an infinite-conductivity line source. However, it is rather difficult to directly establish a mathematical model to describe the pressure drop created by a slanted well in a reservoir based on the infinite-conductivity assumption. Studies have shown that by choosing an appropriate point at which the wellbore pressure response is computed, the wellbore pressure solution of an infinite-conductivity line source can be approximated by the pressure solution of a uniform flux line source. The appropriate calculation point is defined as the equivalentpressure point.

At present, there are two methods of obtaining the bottom hole pressure of the infinite-conductivity inclined well from the response of a uniform flux inclined well. One is to integrate the bottom hole pressure of the uniform flux inclined well along the well trajectory. This method usually needs us to regard the wellbore as a simple line source to avoid doing the surface integral and area-weighted average in order to obtain the bottom hole pressure of the infinite-conductivity inclined well. This is because the pressure of the hole wall will be non-uniform if the influence of well radius is taken into account. This method is inaccurate due to neglecting the influence on pressure of the well radius. In addition, doing the surface integral will affect the computation speed. Another method is the boundary integral method. This method divides the line source well into many discrete line elements and utilizes two relationships to establish an algebraic equation group to obtain the pressure response of the infinite-conductivity inclined well. One relationship is that the pressure on the node of every discrete line element is identical. Another relationship is that the sum of flux of every discrete line element is equal to the total flux.

Cinco (1974) proposed a method to determine the position of the equivalent-pressure point. He regarded the inclined well as a cylinder with a fixed radius and length, not an inclined line source. Then he calculated the pressure at different positions with the aid of a computer to find the point at which the pressure is approximately equal to the average pressure of the whole wall of the cylinder. The found point is called the equivalent-pressure point. Evidently, if the equivalent-pressure point is found, the calculated amount will significantly decrease. With extensive computation Cinco found there are such two points on the elliptic circle which is the intersecting line of any horizontal plane intersecting the inclined well cylinder. The pressure at any one of the two points is approximately equal to the average pressure of the elliptic circle. The two points are located at angles of $90^{\circ}$ and $270^{\circ}$ around the well axis, respectively. For example, the two points are $A^{\prime}$ and $N^{\prime}$ on the top elliptic circle, respectively, and the two points are $D$ and $N$ on the bottom elliptic circle, respectively. In the line $A^{\prime} D$, there are these two points $G$ and $G^{\prime}$ on any one of which the pressure is approximately equal to the average pressure of the line $A^{\prime} D$. So the average pressure of the whole wall of the cylinder can be approximately represented by the pressure at point $G$ or $G^{\prime}$. Point $G$ or $G^{\prime}$ is the equivalent-pressure point that we seek to find. Their positions are showed as follows: 
If $z_{\mathrm{wm}}=0.5 h$, then we have:

$$
z_{\mathrm{wmD}}=\frac{0.5 h}{\frac{L_{\mathrm{w}}}{2}} \sqrt{\frac{k_{\mathrm{h}}}{k_{\mathrm{v}}}}=\frac{h}{L_{\mathrm{w}}} \sqrt{\frac{k_{\mathrm{h}}}{k_{\mathrm{v}}}}
$$

As Fig. 2 illustrates, $B^{\prime} E$ is the axis of the slanted well. $B^{\prime} E=A^{\prime} D=C^{\prime} F=L_{\mathrm{w}}, \angle A^{\prime} B^{\prime} C^{\prime}=\angle A B C=\angle D E F=90^{\circ}$ and $A^{\prime} G^{\prime}=D G=0.3 L_{\mathrm{w}}$. Based on previous studies (Cinco, 1974; Ozkan and Raghavan, 2000) in the cylindrical coordinates, in this paper, if we reason by analogy, the position of the equivalent-pressure point can be determined. The point $G$ or $G^{\prime}$ in Fig. 2 is the equivalent-pressure point, and the coordinates of the equivalent-pressure point are:

$$
\begin{aligned}
& x= \pm 0.3 L_{\mathrm{w}} \sin \theta \\
& y=r_{\mathrm{w}} \\
& z=z_{\mathrm{wm}} \mp 0.3 L_{\mathrm{w}} \cos \theta
\end{aligned}
$$

where $r_{\mathrm{w}}$ is the radius of the inclined well, $\mathrm{m}$.



Fig. 2 Schematic of the equivalent-pressure point of the infinite-conductivity slanted well

The coordinates of the equivalent-pressure point in the distorted space are:

$$
\begin{aligned}
& x_{\mathrm{D}}=\frac{ \pm 0.3 L_{\mathrm{w}} \sin \theta}{L_{\mathrm{w}} / 2}=\frac{ \pm 0.6 L_{\mathrm{w}} \sin \theta}{L_{\mathrm{w}}}= \pm 0.6 \sin \theta \\
& y_{\mathrm{D}}=\frac{r_{\mathrm{w}}}{\frac{L_{\mathrm{w}}}{2}}=r_{\mathrm{wD}} \\
& z_{\mathrm{D}}=\frac{ \pm 0.3 L_{\mathrm{w}} \sin \theta}{\frac{L_{\mathrm{w}}}{2} \tan \theta^{\prime}}+z_{\mathrm{wmD}}=\frac{ \pm 0.6 \sin \theta}{\tan \theta^{\prime}}+z_{\mathrm{wmD}}
\end{aligned}
$$

Combining Eqs. (23)-(25) and Eqs. (12), (17), (18), the bottom hole pressure drawdown $\Delta \bar{p}_{\mathrm{w}}$ can be expressed as follows:

$$
\Delta \bar{p}_{\mathrm{w}}=\Delta \bar{p}_{\mathrm{w} 1}+\Delta \bar{p}_{\mathrm{w} 2}
$$

with

$$
\begin{aligned}
& \Delta \bar{p}_{\mathrm{w} 1}=\frac{c_{1}}{2 s} \int_{-\sin \theta}^{\sin \theta} K_{0}\left[\sqrt{s} \xi\left(x_{\mathrm{wD}}\right)\right] \mathrm{d} x_{\mathrm{wD}} \\
& \Delta \bar{p}_{\mathrm{w} 2}=\frac{c_{1}}{s} \int_{-\sin \theta}^{\sin \theta}\left[\sum_{n=1}^{\infty} K_{0}\left(\alpha_{n} \xi\left(x_{\mathrm{wD}}\right)\right) \cos \beta_{n} \cos \varepsilon_{n}\right] \mathrm{d} x_{\mathrm{wD}} \\
& \xi\left(x_{\mathrm{wD}}\right)=\sqrt{\left(0.6 \sin \theta-x_{\mathrm{wD}}\right)^{2}+r_{\mathrm{wD}}^{2}}
\end{aligned}
$$

\subsection{Computational considerations}

It can be seen from Eqs. (27) and (28) that the integral interval $[-\sin \theta, \sin \theta]$ contains the point $0.6 \sin \theta$. The item $\sqrt{\left(0.6 \sin \theta-x_{\mathrm{wD}}\right)^{2}+r_{\mathrm{wD}}^{2}}$ in the integrand $K_{0}\left[\sqrt{s} \sqrt{\left(0.6 \sin \theta-x_{\mathrm{wD}}\right)^{2}+r_{\mathrm{wD}}^{2}}\right]$ ranging between $r_{\mathrm{wD}}$ and $\sqrt{(1.6 \sin \theta)^{2}+r_{\mathrm{wD}}^{2}}$. It should be noted that a small variation of argument $x$ may cause a big variation of function $K_{0}(x)$. For example, $K_{0}(1) \approx 0.42$, while $K_{0}(50) \approx 3.41 \mathrm{E}-23$ and $K_{0}(100) \approx 4.66 \mathrm{E}-45$. Thus, if we want to obtain results accurate enough with use of the conventional numerical integration method, the integral interval must be divided into many small segments which will cause problems in the computational efficiency of integration. In this case the time that is consumed to compute the wellbore pressure response of slanted wells is far beyond people's tolerance. However, there is very limited published work available on this topic. Some scholars have realized the complexity of the computational issues in computing pressure responses of slanted wells and have done some exploratory investigation, but a satisfactory solution to this problem has not yet been found.

In this paper, according to the characteristics of the integrand in a transient pressure solution for slanted wells, the authors present a computationally efficient algorithm. Concrete steps of the algorithm are as follows:

3.5.1 Partitioning integral interval and variable substitution

The integral interval in Eq. (27) can be partitioned as follows:

$$
\begin{aligned}
\Delta \bar{p}_{\mathrm{w} 1}= & \frac{c_{1}}{2 s} \int_{-\sin \theta}^{0.6 \sin \theta} K_{0}\left[\sqrt{s} \xi\left(x_{\mathrm{wD}}\right)\right] \mathrm{d} x_{\mathrm{wD}} \\
& +\frac{c_{1}}{2 s} \int_{0.6 \sin \theta}^{\sin \theta} K_{0}\left[\sqrt{s} \xi\left(x_{\mathrm{wD}}\right)\right] \mathrm{d} x_{\mathrm{wD}}
\end{aligned}
$$

We choose $0.6 \sin \theta$ as the point of divergence because when $x_{\mathrm{wD}}$ varies from $-\sin \theta$ to $0.6 \sin \theta$ the value of $\left(0.6 \sin \theta-x_{\mathrm{wD}}\right)^{2}$ is monotonously decreasing while when $x_{\mathrm{wD}}$ varies from $0.6 \sin \theta$ to $\sin \theta$ the value of $\left(0.6 \sin \theta-x_{\mathrm{wD}}\right)^{2}$ is continuously increasing. Namely, when $x_{\mathrm{wD}}=0.6 \sin \theta$, the value of $\left(0.6 \sin \theta-x_{\mathrm{wD}}\right)^{2}$ is smallest. Hence we choose $0.6 \sin \theta$ as the point of divergence. 
Let $\tilde{x}_{\mathrm{wD}}=0.6 \sin \theta-x_{\mathrm{wD}}$

$$
\begin{aligned}
\Delta \bar{p}_{\mathrm{w} 1}= & -\frac{c_{1}}{2 s}\left[\int_{1.6 \sin \theta}^{0} K_{0}\left(\sqrt{s} \sqrt{\tilde{x}_{\mathrm{wD}}^{2}+r_{\mathrm{wD}}^{2}}\right) \mathrm{d} \tilde{x}_{\mathrm{wD}}\right. \\
& \left.+\int_{0}^{-0.4 \sin \theta} K_{0}\left(\sqrt{s} \sqrt{\tilde{x}_{\mathrm{wD}}^{2}+r_{\mathrm{wD}}^{2}}\right) \mathrm{d} x_{\mathrm{wD}}\right] \\
= & \frac{c_{1}}{2 s}\left[\int_{0}^{1.6 \sin \theta} K_{0}\left(\sqrt{s} \sqrt{\tilde{x}_{\mathrm{wD}}^{2}+r_{\mathrm{wD}}^{2}}\right) \mathrm{d} \tilde{x}_{\mathrm{wD}}\right. \\
& \left.+\int_{0}^{0.4 \sin \theta} \csc \theta K_{0}\left(\sqrt{s} \sqrt{\tilde{x}_{\mathrm{wD}}^{2}+r_{\mathrm{wD}}^{2}}\right) \mathrm{d} x_{\mathrm{wD}}\right]
\end{aligned}
$$

We define the dimensionless pressures $\bar{p}_{\text {w1D }}, \bar{p}_{\text {w2D }}$ and $\bar{p}_{\text {wD }}$ as follows:

$$
\begin{aligned}
& \bar{p}_{\mathrm{w} 1 \mathrm{D}}=\frac{2 \pi k_{\mathrm{h}} h}{q B \mu} \Delta \bar{p}_{\mathrm{w} 1} \\
& \bar{p}_{\mathrm{w} 2 \mathrm{D}}=\frac{2 \pi k_{\mathrm{h}} h}{q B \mu} \Delta \bar{p}_{\mathrm{w} 2} \\
& \bar{p}_{\mathrm{wD}}=\frac{2 \pi k_{\mathrm{h}} h}{q B \mu} \Delta \bar{p}_{\mathrm{w}}
\end{aligned}
$$

Obviously, the dimensionless bottom hole pressure $\bar{p}_{\mathrm{wD}}$ satisfies

$$
\bar{p}_{\mathrm{wD}}=\bar{p}_{\mathrm{w} 1 \mathrm{D}}+\bar{p}_{\mathrm{w} 2 \mathrm{D}}
$$

Thus

$$
\begin{aligned}
\bar{p}_{\mathrm{wlD}}= & \frac{\csc \theta}{2 s}\left[\int_{0}^{1.6 \sin \theta} K_{0}\left(\sqrt{s} \sqrt{\tilde{x}_{\mathrm{wD}}^{2}+r_{\mathrm{wD}}^{2}}\right) \mathrm{d} \tilde{x}_{\mathrm{wD}}\right. \\
& \left.+\int_{0}^{0.4 \sin \theta} K_{0}\left(\sqrt{s} \sqrt{\tilde{x}_{\mathrm{wD}}^{2}+r_{\mathrm{wD}}^{2}}\right) \mathrm{d} \tilde{x}_{\mathrm{wD}}\right]
\end{aligned}
$$

Similarly, we can get the following expression:

$$
\begin{aligned}
& \Delta \bar{p}_{2}=\frac{c_{1}}{s} \int_{0}^{1.6 \sin \theta}\left[\sum_{n=1}^{\infty} K_{0}\left(\breve{\xi}\left(\tilde{x}_{\mathrm{wD}}\right) \alpha_{n}\right) \cos \beta_{n} \cos \gamma_{n}\right] \mathrm{d} \tilde{x}_{\mathrm{wD}} \\
& +\frac{c_{1}}{s} \int_{0}^{0.4 \sin \theta}\left[\sum_{n=1}^{\infty} K_{0}\left(\breve{\xi}\left(\tilde{x}_{\mathrm{wD}}\right) \alpha_{n}\right) \cos \beta_{n} \cos \gamma_{n}^{\prime}\right] \mathrm{d} \tilde{x}_{\mathrm{wD}}
\end{aligned}
$$

with

$$
\begin{aligned}
& \breve{\xi}\left(\tilde{x}_{\mathrm{wD}}\right)=\sqrt{\tilde{x}_{\mathrm{wD}}^{2}+r_{\mathrm{wD}}^{2}} \\
& \gamma_{n}=\frac{\frac{0.6 \sin \theta-\tilde{x}_{\mathrm{wD}}}{\tan \theta^{\prime}}+z_{\mathrm{wmD}}}{h_{\mathrm{D}}} \\
& \gamma_{n}^{\prime}=\frac{\frac{0.6 \sin \theta+\tilde{x}_{\mathrm{wD}}}{\tan \theta^{\prime}}+z_{\mathrm{wmD}}}{h_{\mathrm{D}}}
\end{aligned}
$$

With the definition of dimensionless pressure $\bar{p}_{\text {w2D }}$, we have



$$
\begin{aligned}
& +\frac{\csc \theta^{0.4 \sin \theta}}{s} \int_{0}^{n}\left[\sum_{n=1}^{\infty} K_{0}\left(\breve{\xi}\left(\tilde{x}_{\mathrm{wD}}\right) \alpha_{n}\right) \cos \beta_{n} \cos \gamma_{n}^{\prime}\right] \mathrm{d} \tilde{x}_{\mathrm{wD}}
\end{aligned}
$$

\subsubsection{Numerical integration with variable step sizes}

First, the integral intervals $[0,1.6 \sin \theta]$ and $[0,0.4 \sin \theta]$ are partitioned into $N$ small segments with variable step sizes. $r_{0}$, $r_{1}, r_{2}, r_{3}, \ldots$, and $r_{N}$ denote the $N+1$ end points created by the partition.

Obviously, $r_{0}=0$ and $r_{N}=1.6 \sin \theta$ (or $0.4 \sin \theta$ ).

Let

$$
\left\{\begin{array}{l}
r_{1}=a_{0} \\
r_{2}=r_{1} d=a_{0} d \\
r_{3}=r_{2} d=a_{0} d^{2} \\
\quad \vdots \\
r_{N}=r_{N-1} d=a_{0} d^{N-1}
\end{array}\right.
$$

where $d$ is the common ratio.

Given a constant $a_{0}$, then we have

$$
\frac{r_{N}}{a_{0}}=d^{(N-1)}
$$

The logarithm expression of Eq. (39) is

$$
\ln \left(\frac{r_{N}}{a_{0}}\right)=(N-1) \ln (d)
$$

So we can obtain

$$
d=\exp \left[\ln \left(\frac{r_{N}}{a_{0}}\right) /(N-1)\right]
$$

If the total number $(N)$ of small segments is given, the value of $d$ can be computed with Eq. (41), thus the value of $r_{i}$ can be calculated. Then we have

$$
\int_{r_{0}}^{r_{N}} f\left(\tilde{x}_{\mathrm{wD}}\right) \mathrm{d} \tilde{x}_{\mathrm{wD}}=\sum_{i=0}^{N-1} \int_{r_{i}}^{r_{i+1}} f\left(\tilde{x}_{\mathrm{wD}}\right) \mathrm{d} \tilde{x}_{\mathrm{wD}}
$$

In each small integral segment $\left[r_{i}, r_{i+1}\right]$, the GaussLegendre, Simpson or Runge-Kutta methods can be employed to calculate the integration. Eqs. (35) and (37) can be efficiently computed with the algorithm proposed in this paper, then the dimensionless bottom hole pressure of slanted 
wells can he ohtained with Eq. (34).

Calculation practice has shown that the total number of the small integral segments (i.e. $n$ ) partitioned by the variable step-size numerical integration proposed in this paper is much less than that of the equal step-size numerical integration with the same accuracy. The algorithm proposed in this paper can greatly save the computational time and the computational accuracy is also improved.

\subsubsection{Comparison of the computation amount}

In order to verify the computational accuracy and efficiency of the method called piecewise integration with variable step sizes proposed in this paper, we compared it with the conventional piecewise integration with equal step sizes. The parameters contrasted include the segmentation amount, the number of times for calculating the numerical integration and the total time consumed in calculation, etc. Here we choose a horizontal well for computation and comparison because the horizontal well is a particular inclined well whose pressure response is familiar to people.

Fig. 3a and Fig. 3b show a comparison of typical curves calculated by the two methods. Fig. 3a indicates that when the variable step-size piecewise integration is used and the number of segmentations " $n_{1}$ " only equals 4 , the computational accuracy is already high enough. However when the equal step-size piecewise integration is used and the number of segmentations " $n_{1}$ " equals 4 , the computational accuracy is very low, and when the number of segmentations " $n$," equals 8 the computational accuracy is improved a little but is still low. Fig. 3b shows that for the equal step-size piecewise integration, when the number of segmentations " $n_{1}$ "reaches 16 , the accuracy is comparative to the one when the number of segmentations " $n_{1}$ " is 4 for the variable stepsize piecewise integration. The comparison of computational results by the two methods is listed in Table 1. From Table 1 we can see the number of segmentations and the number of times for calculating the numerical integration by this method proposed in this paper is only $1 / 4$ of which by the conventional method, and the total time consumed in calculation is only $1 / 3.7$ of which consumed in calculation by the conventional method, so the variable step-size piecewise integration proposed in this paper may significantly reduce the computational amount and save a great deal of time.

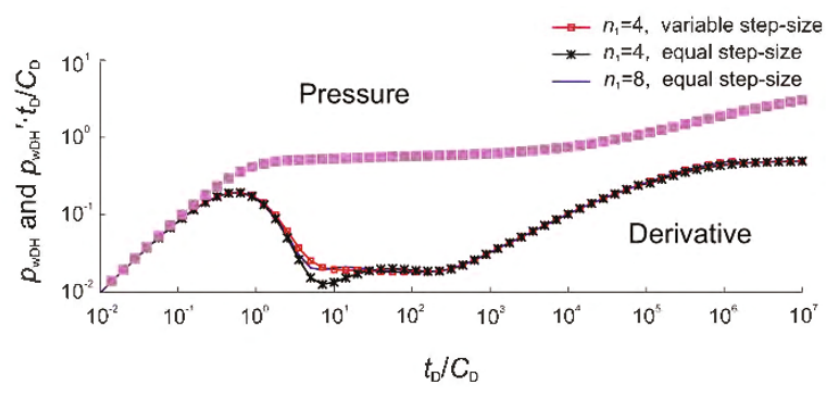

Fig. 3a A comparison of computational results obtained by the variable step-size piecewise integration and the equal step-size piecewise integration, respectively $\left(C_{\mathrm{D}}=20, S=0.5, h=20 \mathrm{~m}, L_{\mathrm{w}}=800 \mathrm{~m}, k_{\mathrm{v}} / k_{\mathrm{h}}=0.6\right.$, $\left.r_{\mathrm{w}}=0.1 \mathrm{~m}, \theta=90^{\circ}\right)$

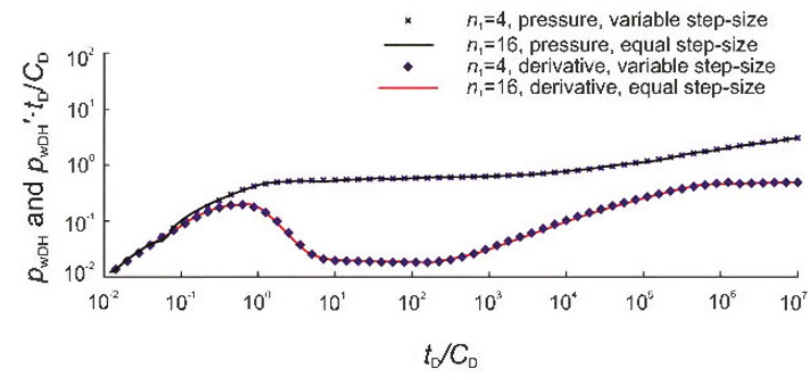

Fig. 3b A comparison of computational results obtained by the variable step-size piecewise integration and the equal step-size piecewise integration, respectively $\left(C_{\mathrm{D}}=20, S=0.5, h=20 \mathrm{~m}, L_{\mathrm{w}}=800 \mathrm{~m}\right.$, $k_{\mathrm{v}} / k_{\mathrm{h}}=0.6, r_{\mathrm{s}}=0.1 \mathrm{~m}, 0=90^{\circ}$ )

Table 1 A comparison of computation amount and total time consumed in calculation by the variable step-size piecewise integration and the equal stepsize piecewise integration, respectively

\begin{tabular}{c|ccccccc}
\hline Computational method & $n_{1}$ & $n_{2}$ & $n_{3}$ & $n_{4}$ & $n_{5}$ & $n_{6}$ & $T, s$ \\
\hline Variable step-size integration & 4 & 60 & 10 & 8 & 2640 & 21120 & $\approx 0.88$ \\
Equal step-size integration & 16 & 60 & 10 & 8 & 10560 & 84480 & $\approx 3.22$ \\
\hline Ratio & $1: 4$ & $1: 1$ & $1: 1$ & $1: 1$ & $1: 4$ & $1: 4$ & $\approx 1: 3.7$ \\
\hline
\end{tabular}

In this paper $n_{1}$ is the total number of segmentations along the inclined well, $n_{1}=2 N ; n_{2}$ is the number of the calculated points; $n_{3}$ is the sum of terms practically selected in the infinite series of Eq. (37) when calculating; $n_{4}$ is the sum of terms selected in the series of Stehfest numerical inversion equation; $n_{5}$ is the total number of times of the integration when $n_{2}$ points are calculated (not by numerical inversion), $n_{5}=\left(2 N+2 N \times n_{3}\right) \times n_{2} ; n_{6}$ is the total number of times of the integration when $n_{2}$ points are calculated (by numerical inversion $), n_{6}=\left(2 N+2 N \times n_{3}\right) \times n_{2} \times 8 ; N$ is the number of segmentations along the half length of the inclined well; $T$ is the total time consumed in calculation.

\subsection{Consideration of wellbore storage and skin effects}

The dimensionless bottom hole pressure $\bar{p}_{\mathrm{wD}}$ mentioned above is derived under the assumption of no wellbore storage and skin effects, while the effects of wellbore storage and skin factor can be easily incorporated in the pressure solution with Eq. (43) which was given by Van Everdingen and Hurst (1949). The expression is derived by combining Duhamel's principle and the definitions of wellbore storage coefficient and skin factor.

$$
\bar{p}_{\mathrm{wDH}}=\frac{s \bar{p}_{\mathrm{wD}}+S}{s+C_{\mathrm{DL}} s^{2}\left(s \bar{p}_{\mathrm{wD}}+S\right)}
$$

where $\bar{p}_{\text {wDI }}$ and $\bar{p}_{\text {wD }}$ are the dimensionless bottom hole pressure which takes or not the wellbore storage and skin factor into account, respectively; $S$ is the skin factor; $C_{\mathrm{DL}}$ is the dimensionless wellbore storage coefficient, $C_{\mathrm{DL}}$ is expressed as follows:

$$
C_{\mathrm{DL}}=\frac{C}{2 \pi h \phi C_{\mathrm{t}} L^{2}}
$$


where $C$ is the wellbore storage coefficient, $\mathrm{m}^{3} / \mathrm{Pa} ; C_{\mathrm{t}}$ is the total compressibility, $\mathrm{Pa}^{-1} ; L$ is the reference length, $\mathrm{m}$, and here $L=0.5 L_{\mathrm{w}}$ according the definition of $L$ in the section before Eq. (17).

When the angle of inclination varies the range of $L_{\mathrm{w}}$ also will vary. Eq. (44) indicates that $C_{\mathrm{DL}}$ is the function of the reference length $L$ (i.e. $0.5 L_{\mathrm{w}}$ ). To make sure the dimensionless wellbore storage coefficient has the same expression under different well length $L_{\mathrm{w}}$ so that it be able to be measured in the same scale, here we define another dimensionless wellbore storage coefficient $C_{\mathrm{D}}$.

$$
C_{\mathrm{D}}=\frac{C}{2 \pi h \phi C_{\mathrm{t}} r_{w}{ }^{2}}
$$

From Eq. (45) we may see the definition of the dimensionless wellbore storage coefficient $C_{\mathrm{D}}$ is based on the well radius $r_{\mathrm{w}}$, bnt not the half-length $0.5 L_{\mathrm{w}}$ of the inclined well. The definition of $C_{\mathrm{D}}$ is same with the traditional definition of dimensionless wellbore storage coefficient for vertical wells.

With Eq. (44) and Eq. (45), we obtain

$$
C_{\mathrm{DL}}=C_{\mathrm{D}}\left(\frac{r_{\mathrm{w}}}{L}\right)^{2}=C_{\mathrm{D}} r_{\mathrm{wD}}^{2}
$$

Eq. (43) is transformed into the following expression:

$$
\bar{p}_{\mathrm{wDH}}=\frac{s \bar{p}_{\mathrm{wD}}+S}{s+r_{\mathrm{wD}}^{2} C_{\mathrm{D}} s^{2}\left(s \bar{p}_{\mathrm{wD}}+S\right)}
$$

\section{Analyses of type curves and flow mechanisms}

The type curves of slanted wells with any inclination angle in real space can be plotted with use of the Stehfest numerical inversion algorithm (Stehfest, 1970). Calculation practice has proved that the method proposed in this paper can significantly save the computation time and improve the computational accuracy.

Here we present some results to show the accuracy, efficiency and the practical use of the algorithm mentioned above.

When drawing the typical curves, the $X$-coordinate is $t_{\mathrm{D}} /$ $C_{\mathrm{D}}, Y$-coordinate is the dimensionless pressure $p_{\mathrm{wDH}}$ or the dimensionless pressure derivative $p_{\mathrm{wDH}} \cdot t_{\mathrm{D}} / C_{\mathrm{D}}$.

In order to avoid the influence of the angle of inclination and the inclined well length, the dimensionless time $t_{\mathrm{D}}$ also is defined based on the well radius $r_{\mathrm{w}}$. The definition of $t_{\mathrm{D}}$ is as follows:

$$
t_{\mathrm{D}}=\frac{k_{\mathrm{h}} t}{\phi \mu C_{\mathrm{t}} r_{\mathrm{w}}{ }^{2}}
$$

However from Eq. (2) we may see that the Laplace transformation of the pressure solution in this paper is with respect to $t_{\mathrm{DL}}$. So $p_{\mathrm{wDH}}$ and $p_{\mathrm{wDH}} \cdot t_{\mathrm{D}} / C_{\mathrm{D}}$ which are calculated directly by numerical inversion is corresponding to $t_{\mathrm{DL}}$. It seems that $t_{\mathrm{DL}}$ creates a difficulty for us, but in practice the difficulty can be overcome. The method of resolving it is as follows:

According previous definitions, we obtain the following relationship between $t_{\mathrm{D}} / C_{\mathrm{D}}$ and $t_{\mathrm{DL}}$ :

$$
t_{\mathrm{DL}}=\left(\frac{r_{\mathrm{w}}}{L}\right)^{2} \cdot\left(t_{\mathrm{D}} / C_{\mathrm{D}}\right) \cdot C_{\mathrm{D}}={r_{\mathrm{wD}}}^{2} \cdot\left(t_{\mathrm{D}} / C_{\mathrm{D}}\right) \cdot C_{\mathrm{D}}
$$

When giving the value of $t_{\mathrm{D}} / C_{\mathrm{D}}$, the value of $t_{\mathrm{DL}}$ may be obtained with Eq. (49). Then by numerical inversion, the values of $p_{\mathrm{wDH}}$ and $p_{\mathrm{wDH}} \cdot t_{\mathrm{D}} / C_{\mathrm{D}}$ responding to $t_{\mathrm{DL}}$ may be obtained. So the values of $p_{\mathrm{wDH}}$ and $p_{\mathrm{wDH}} \cdot t_{\mathrm{D}} / C_{\mathrm{D}}$ responding to $t_{\mathrm{D}} / C_{\mathrm{D}}$ may be obtained. The difficulty is overcome immediately.

\subsection{Several special cases and comparison of pressure responses calculated by two methods}

\subsubsection{Fully penetrating vertical well $\left(\theta \rightarrow 0^{\circ}\right.$ and $\left.L_{\mathrm{w}}=\boldsymbol{h}\right)$}

The model proposed in this paper cannot be numerically evaluated for vertical wells (i.e. $\theta=0^{\circ}$ ). It is, however, possible to obtain the pressure responses of vertical wells by letting $\theta=\varepsilon$, while $\varepsilon$ is sufficiently small. $L_{\mathrm{w}}$ equals $h$ for vertical wells. Fig. 4 shows a comparison of pressure responses of a vertical well calculated by the method proposed in this paper and with commercial software, respectively. Fig. 4 shows that the flow regimes identified in the transient response are wellbore storage, transitional flow, and radial flow. The results obtained by the two methods match. This indicates that for vertical wells the pressure response calculated by the method proposed in this paper is very reasonable and reliable.

Though the pressure responses compared here are for vertical wells, the method proposed in this paper used the same pressure solution and employed the same means of numerical integration for vertical, horizontal and inclined wells, the calculation method does not change as the angle of inclination varies, and so the calculation accuracy will not be influenced by the change of angle of inclination. Namely since the calculation accuracy is very high for vertical wells, the calcnlation accuracy will still be high for those wells with any angle of inclination.

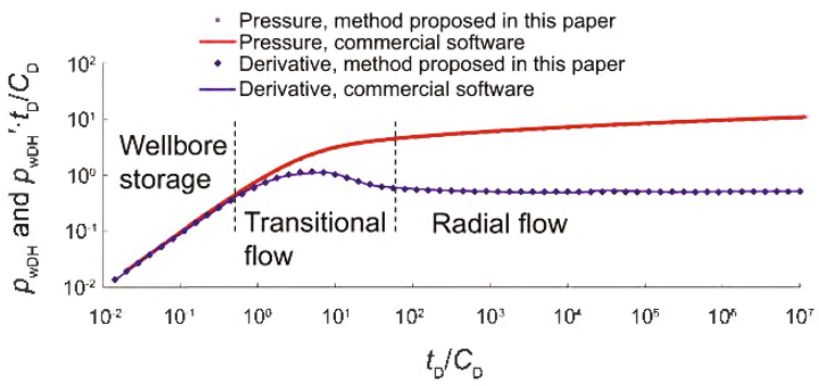

Fig. 4 A comparison of the pressure responses of a vertical well calculated by the method proposed in this paper and the commercial softwarc, respectively $\left(L_{\mathrm{w}}=h=20 \mathrm{~m}, C_{\mathrm{D}}=20, S=0.5, k_{\mathrm{v}} / k_{\mathrm{h}}=0.6, r_{\mathrm{w}}=0.1 \mathrm{~m}\right.$, $\left.0=\varepsilon \rightarrow 0^{\circ}\right)$

\subsubsection{Horizontal well $\left(\theta=90^{\circ}\right)$}

Fig. 5 shows a comparison of the pressure responses of a horizontal well calculated by the method proposed in this paper and the commercial software, respectively. 
Fig. 5 indicates that the results obtained by the two methods are in good agreement. This indicates that for horizontal wells the pressure responses calculated by the method proposed in this paper is also reasonable and reliable. The flow regimes (Fig. 5) include: (1) a pure wellbore storage period whose pressure and derivative curves are straight lines of unit slope; (2) a transitional flow period whose pressure derivative curve appears a "hump"; (3) an early radial flow period whose pressure derivative curve is a horizontal line; (4) an intermediate linear flow period whose pressure and derivative curves have the common slope of " 0.5 "; (5) a late pseudo-radial flow period, and in this period the pressure derivative curve is a horizontal line of which the height is 0.5 .

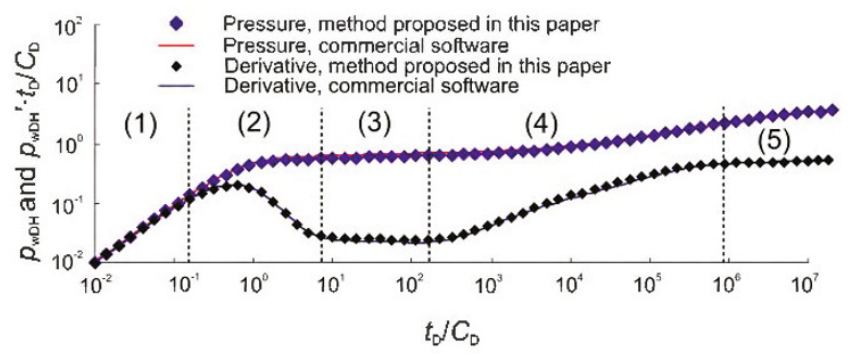

Fig. $5 \mathrm{~A}$ comparison of the pressure responses of a horizontal well calculated by the method proposed in this paper and with commercial software, respectively $\left(C_{\mathrm{D}}=20, S=0.5, h=20 \mathrm{~m}, k_{\mathrm{v}} /\right.$ $k_{\mathrm{h}}=0.6, r_{\mathrm{w}}=0.1 \mathrm{~m}, L=600 \mathrm{~m}, \theta=90^{\circ}$ )

Fig. 6 shows the effect of $h_{\mathrm{D}}$ on pressure responses of horizontal wells obtained by our algorithm. The bigger the value of $h_{\mathrm{D}}$ is, the higher the pressure derivative curve in the early radial flow period is, and the height of pressure derivative curve is about $h_{\mathrm{D}} / 4$. The results obtained by our algorithm are in good agreement with those published in the literature (Ozkan et al, 1991a; 1991b; 2000).

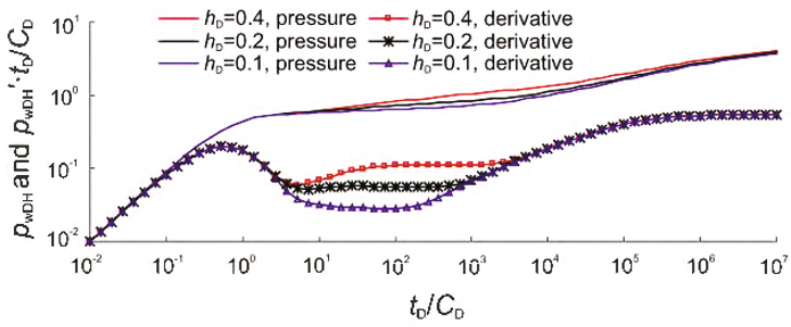

Fig. 6 Effect of $h_{\mathrm{D}}$ on pressure responses of horizontal wells obtained by the algorithm proposed in this paper $\left(C_{D}=20, S=0.5\right.$, $r_{\mathrm{WD}}=0.0005, Z_{\mathrm{wmD}}=0.5 h_{\mathrm{D}}, \theta=90^{\circ}$ )

\subsubsection{Partially penetrating vertical well $\left(\theta \rightarrow 0^{\circ}\right.$ and $\left.0<L_{\mathrm{w}}<h\right)$}

Fig. 7 shows a comparison of the pressure responses of a partially penetrating vertical well calculated by the method proposed in this paper and the source function method, respectively, and the perforation degree $L_{\mathrm{w}} / h$ is 0.5 . From Fig. 7 we see there is an apparent spherical flow period in the whole flow process of a partially penetrating vertical well. The pressure derivative curve in the spherical flow period is a straight line of a slope of -0.5 , which reflects that the well is partially penetrated.

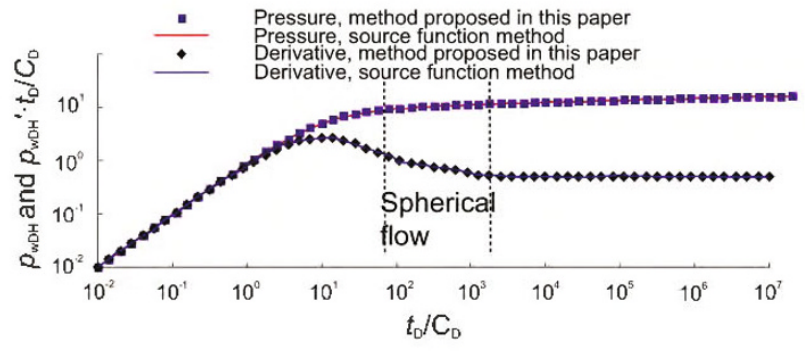

Fig. 7 A comparison of the pressure responses of a partially penetrating vertical well calculated by the method proposed in this paper and by source function method, respectively $\left(C_{\mathrm{D}}=20, S=1, h=20 \mathrm{~m}, L_{\mathrm{w}}=h_{\mathrm{w}}=10 \mathrm{~m}, k_{\mathrm{v}} / k_{\mathrm{h}}=0.1\right.$, $\left.r_{\mathrm{w}}=0.1 \mathrm{~m}, \theta=\varepsilon \rightarrow 0, \theta \rightarrow 0^{\circ}, L_{\mathrm{w}} / h=0.5\right)$

The above comparison and analysis proved that the calculation accuracy of the method proposed in this paper is high, and the calculation results are also reliable.

\subsection{Slanted wells with any inclination angle $\left(0^{\circ}<\theta<90^{\circ}\right.$ and $0<L_{\mathrm{w}}<\boldsymbol{h} / \cos \theta$ )}

Fig. 8 shows a comparison of the pressure responses of slanted wells with an inclination angle of $85^{\circ}$ obtained by the algorithm proposed in this paper and commercial software, respectively.

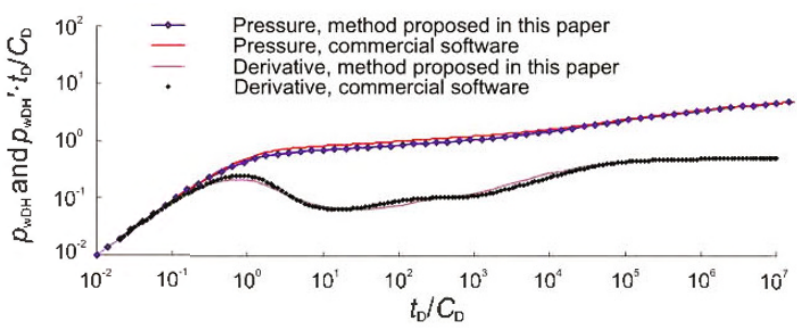

Fig. 8 A comparison of the pressure responses of an inclined well calculated by the method proposed in this paper and a commercial software package, respectively $\left(C_{\mathrm{D}}=20, S=0.5, h=20 \mathrm{~m}, L_{\mathrm{w}}=229 \mathrm{~m}, k_{\mathrm{v}} / k_{\mathrm{h}}=0.6, r_{\mathrm{w}}=0.1 \mathrm{~m}, 0=85^{\circ}\right)$

The comparison indicates that: 1) The pressure response curves obtained by both methods have similar features. In the early period, there is a short radial flow period followed by a long transitional flow behavior and then a linear flow at later times. 2) The curves in the transitional and linear flow periods obtained by the two means have some minor difference. In the previous discussion we had already made some analysis and known that though the pressure responses compared here are for vertical wells, the method proposed in this paper used the same pressure solution and employed the same means of numerical integration for vertical, horizontal and inclined wells, the calculation method does not change as the inclination angle varies, and so the calculation accuracy will not be significantly influenced by the change of the inclination angle. Namely since the calculation accuracy is very high for vertical wells, the calculation accuracy is still high for those wells with any inclination angle. Hence there is some difference in that the accuracy of the method proposed in this paper is higher than that of the commercial software. In addition, the commercial software is not able to compute the pressure response of inclined wells with $85^{\circ}-90^{\circ}$ inclination 
angle, but the method proposed in this paper is able to compute it and is more flexible than the commercial software.

Fig. 9(a) is the pressure responses of inclined wells with $70^{\circ}-90^{\circ}$ inclination angle calculated by the method proposed in this paper.

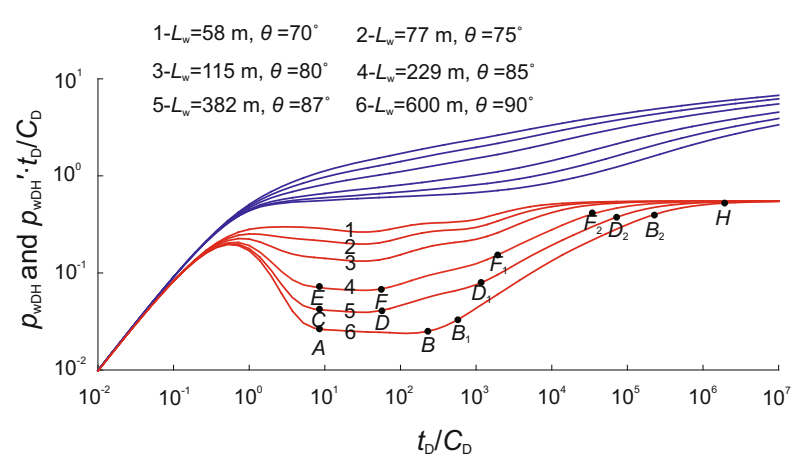

Fig. 9a Pressure responses of inclined wells with $70^{\circ}-90^{\circ}$ inclination angle calculated by the method proposed in this paper ( $C_{\mathrm{D}}=20, S=0.5, h=20 \mathrm{~m}, k_{\mathrm{v}}$ / $\left.k_{\mathrm{h}}=0.6, r_{\mathrm{w}}=0.1 \mathrm{~m}\right)$

There are six groups of curves in Fig. 9a and each of them corresponds to a different inclination angle and well length. From top to bottom, the inclination angle increases from $70^{\circ}$ to $90^{\circ}$. When the inclination angle $\theta$ equals $90^{\circ}$, the transitional flow period between the early radial flow and the linear flow is the shortest. When $\theta$ is much smaller than $90^{\circ}$ the transitional flow period apparently becomes long. For example, both $D D_{1}$ and $F F_{1}$ are longer than $B B_{1}$. The smaller the $\theta$ is, the shorter the linear flow period is, for example $F_{1} F_{2}<D_{1} D_{2}<B_{1} B_{2}$.

Fig. $9 \mathrm{~b}$ is the pressure responses of inclined wells with $0^{\circ}-60^{\circ}$ inclination angle calculated by the method proposed in this paper. When $\theta$ is smaller than $60^{\circ}$ the linear flow period is indistinct, and when $\theta$ is close to $0^{\circ}$ the radial flow in the early period and the linear flow in the middle period will vanish completely to display a single radial flow period in the late period because the radial flow direction in the early period is the same as the flow direction of the radial flow in the late period. In the late radial flow period, the pressure derivative displays a horizontal line of height 0.5 .

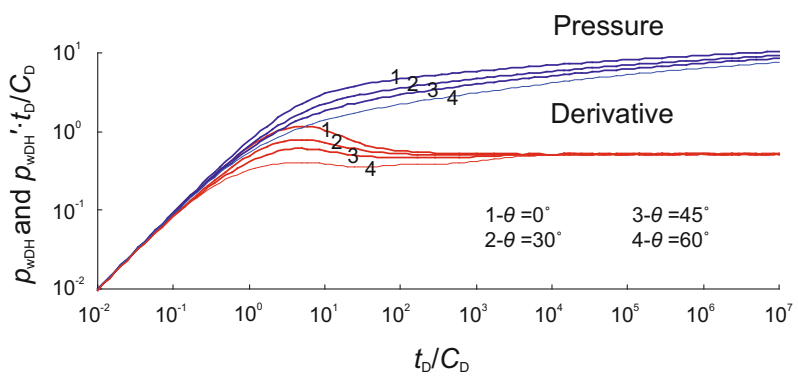

Fig. 9b Pressure responses of inclined wells with $0^{\circ}-60^{\circ}$ inclination angle calculated by the method proposed in this paper

Fig. 10 shows the influence of inclination angle on the pressure response of inclined wells with the same well length. From Fig. 10 we see that the smaller the inclination angle is, the shorter the early radial flow period is, such as $A B_{1}<A B_{2}<A B_{3}$, and the longer the transitional flow period between the early radial flow and the linear flow is, such as $B_{1} C>B_{2} C>B_{3} C$.

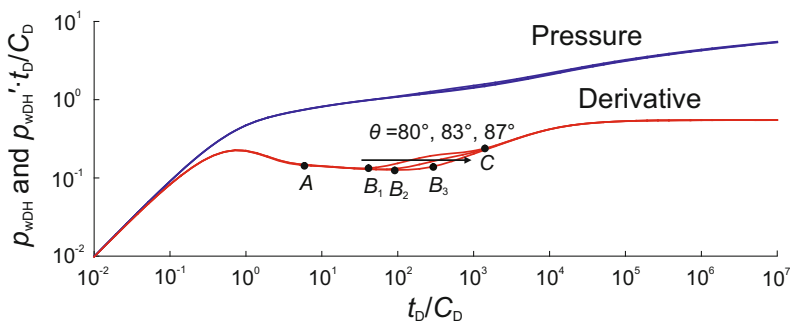

Fig. 10 Influence of the inclination angle on the pressure response of inclined wells with the same well length $\left(L_{\mathrm{w}}=115 \mathrm{~m}, C_{\mathrm{D}}=20, S=0.5, h=20\right.$ $\left.\mathrm{m}, k_{\mathrm{v}} / k_{\mathrm{h}}=0.6, r_{\mathrm{w}}=0.1 \mathrm{~m}\right)$

\section{Conclusions}

1) Based on rigorous derivation, a transient pressure solution for slanted wells is presented. Assuming an infiniteconductivity wellbore, the location of the equivalent-pressure point is determined.

2) According to the characteristics of the integrand in the transient pressure solution for slanted wells, the whole integral interval is partitioned into several small integral intervals, and then the method of variable substitution and the variable-step size numerical integration are employed. The amount of computation is greatly reduced and the computational efficiency is greatly improved. The algorithm proposed in this paper thoroughly solved the difficulty in the efficient and high-speed computation of transient pressure distribution of slanted wells with any inclination angle.

3) Several special cases are computed by the model and the efficient algorithm proposed in this paper, such as horizontal wells, fully penetrating vertical wells and partially penetrating vertical wells. The results are compared with those published in the literature and are in good agreement. In addition, the pressure responses of slanted wells with different inclination angles are computed and analyzed. Results show that the model and the algorithm proposed in this paper is computationally efficient with high accuracy.

Note: The authors can provide the efficient calculation program written in Visual Basic if required.

\section{Acknowledgements}

The authors are grateful for financial support from the special fund of China's central government for the development of local colleges and universities-the project of national first-level discipline in Oil and Gas Engineering, the National Science Fund for Distinguished Young Scholars of China (Grant No. 51125019) and the National Program on Key fundamental Research Project (973 Program, Grant No. 2011CB201005).

\section{References}

Besson J. Performance of slanted and horizontal wells in an anisotropic 
medium. Paper SPE 20965 presented at the European Petroleum Conference, 21-24 October 1990, The Hague

Chen G, Tehrani D H and Peden J M. Pressure distribution created by a slanted well with elliptic inner boundary condition. Paper SPE 29121 presented at the SPE Symposium on Reservoir Simulation, 12-15 February 1995, San Antonio

Cinco H. Unsteady-state pressure distributions created by a slanted well or a well with an inclined fracture. Ph.D Dissertation. Stanford University, Stanford, California. 1974

Cinco H, Miller F G and Ramey Jr H J. Unsteady-state pressure distribution created by a directionally drilled well. Journal of Petroleum Technology. 1975a. 27(11): 1392-1400

Cinco H, Ramey Jr H J and Miller F G. Pseudoskin factors for partially penetrating, directionally drilled wells. Paper SPE 5589 presented at the Fall Meeting of the Society of Petroleum Engineers of AIME, 28 September-1 October, 1975b, Dallas

Fair P S. Horizontal well pressure transient analysis for Gulf of Mexico reservoirs (adapting the slant well solution to layered media). SPE 104480, 2008

Harmohan G, Rayed A, Mohammed BI, et al. Pressure transient behavior of horizontal and slant wells intersecting a high permeability layer. Paper SPE 105616 presented at the SPE Middle East Oil and Gas Show and Conference, 11-14 March 2007, Kingdom of Bahrain

Khattab H A, Yeh N S and Agarwd R G. Pressure transient behavior of slanted wells in single and multiple-layered systems. Paper SPE 22730 presented at the SPE Annual Technical Conference and Exhibition, 6-9 October 1991, Dallas

Liao X W. Discussion of a slanted well test model in dual porosity reservoirs with pseudo steady state flow. Petroleum Explorationand Development. 1998. 25(5): 57-61 (in Chinese)
Li W, Lu D T, Wang L, et al. A transient pressure solution for inclined wells with complex boundaries. Well Testing. 2009. 18(6): 1-5 (in Chinese)

$\mathrm{Lu}$ P. Horizontal and slanted wells in layered reservoirs with crossflow. MS Thesis. Stanford University, Stanford, California. 1997

Ozkan E and Raghavan R. New solutions for well-test-analysis problems: Part 1-Analytical considerations. SPEFE. 1991a. 6(3): 359-368

Ozkan E and Raghavan R. New solutions for well-test-analysis problems: Part 2-Computational considerations and applications. SPEFE. 1991b. 6(3): 369-378

Ozkan E and Raghavan R. A computationally efficient transient-pressure solution for inclined wells. SPE Reservoir Evaluation\& Engineering. 2000. 3(5): 414-425

Stehfest H. Numerical inversion of Laplace transform. Communications of the ACM. 1970. 13(1): 47-49

Van Everdingen A F and Hurst W. The application of the Laplace transformation to flow problems in reservoirs. Transaction of American Institute of Mining, Metallurgical, and Petroleum Engineers (Trans. AIME). 1949. 186: 305-324

Wang D S, Nie L X, Li Z M. Study of the analytic method for the well testing of deflecting wells. Journal of Xi' an Shiyou University (Natural Science Edition). 2006. 21(5): 51-54 (in Chinese)

Wang D S, Nie L X, Li Z M. Well test analysis by using a well drawdown curve in a multiple-deviated well system. Journal of Oil and Gas Technology. 2006. 28(1): 95-97 (in Chinese)

Wang D S, Zhang L, Nie L X, et al. A computationally efficient transient pressure solution for an inclined multiwell system. Journal of Hydrodynamics. 2005. 20(4): 527-530 (in Chinese)

(Edited by Sun Yanhua) 